\title{
Circus2CSP: A Tool for Model-Checking Circus Using FDR
}

\author{
Artur Oliveira Gomes ${ }^{1}$ and Andrew Butterfield ${ }^{2}$ \\ 1 Universidade Federal de Mato Grosso do Sul, Brazil \\ artur.gomes@ufms.br \\ 2 School of Computer Science and Statistics \\ Trinity College Dublin, Ireland \\ butrfeld@tcd.ie
}

\begin{abstract}
In this paper, we introduce Circus2CSP, a tool that automatically translates Circus into $C S P_{M}$, with an implementation based on a published manual translation scheme. This scheme includes new and modified translation rules that emerged as a result of experimentation. We addressed issues with FDR state-space explosion, by optimising our models using the Circus Refinement Laws. We briefly describe the usage of Circus2CSP along with a discussion of some experiments comparing our tool with the literature.
\end{abstract}

\section{Introduction}

Among the range of verification techniques, model checking is used for exploring all the possible states a reactive system can reach. In other words, the algorithms used for model-checking will evaluate all possible scenarios of a system, which may eventually reach an undesired one, depending on the property that is being evaluated. Moreover, the focus of model-checking is on the system's behaviour rather than how the model would manage its data. Therefore, a system whose behaviour strongly relies on its data may become difficult to check, since the data may range over infinite domains.

There has been an effort from the community in order to design a systematic approach for model-checking Circus, which due to its combination of formalisms, is quite a challenge. Circus 34 is a formal language that combines structural aspects of a system using the Z language [36] and the behavioural aspects using CSP [32, along with the refinement calculus 22] and Dijkstra's guarded commands [7. Its semantics is based on the Unifying Theories of Programming (UTP) [15. As an initial attempt to model-check Circus, we participated in the ABZ'16 haemodialysis case study [12, producing a Circus specification, manually translating it into $C S P_{M}$, which we then checked with FDR 9. Moreover, when translating Circus into CSP, we adapted the Circus model to map the structural $\mathrm{Z}$ parts into appropriate CSP.

Unlike in Circus processes, an explicit notion of state variables is not present in CSP processes. Therefore, in order to translate Circus state, we would either 
translate it into a memory process $23 / 17 / 29]$, allowing other processes to read and write the values by synchronising on memory 'get' and 'put' events, or to transform the state variables into process parameters, as used by Beg [4. For instance, we captured the state-based features of Circus in CSP using a memory process synchronising on channels for reading and updating the values of the state variables. Such an approach was also used while model checking [10 the ARINC 653 [2] architecture.

In this paper, we present Circus2CSP ${ }^{3}[13$, a tool capable of model-checking specifications designed in Circus using FDR. It was developed by extending JAZA [33, a Z animator written in Haskell, in order to cover the Circus abstract syntax. The rest of the paper is organized as follows: In Sec. 2, we discuss the main goal of this work. A brief description of some experiments using Circus2CSP is presented in Sec. 3. The paper is concluded in Sec. 4 .

\section{Circus2CSP: Requirements and Goals}

Our translation is based on that developed by Oliveira in the Compass project [26]27, which is based on repeated application of carefully selected Circus refinement laws, all of which happen to be equivalences. Such a translation uses set of rules for refining state-rich Circus into stateless processes that can be mapped into $C S P_{M}$.

Our focus while model-checking Circus is to produce a model in $C S P_{M}$ where FDR can evaluate using as little computing resources as possible. As such, we provide a refined model from the strategy presented by Oliveira et al. [26], where our tool is capable of producing $C S P_{M}$ models from larger specifications and making it possible for model-checking them using FDR. We highlight that because FDR is a refinement checker, it is not possible to perform temporal logic checks, which is further discussed by Lowe [20].

The entire toolset is developed as an extension of JAZA, which parses Z specifications written in $\mathrm{AT}_{\mathrm{E} X} \mathrm{X}$, the same input used by the Community $\mathrm{Z}$ Tools. Our goal was to produce a framework using the infrastructure available from JAZA, where the parser for $\mathrm{Z}$ was extended and now supports Circus and from there, we include new modules like the translation tool and the refinement calculator for Circus. Moreover, our tool is linked to FDR, and may also be integrated with other tools in the future. Our contribution here is mainly related to the fulfilment of a tool for automatically model-checking Circus.

The reason we adopted the translation presented by Oliveira et al., is that, even though it is a manual translation, with no tool support involved, each translation step is justified by the Circus refinement laws, which have been formally proved to be correct. Currently, their approach covers a subset of Circus. However, our investigation 14 through experiments with the implementation of such rules demonstrated that such an initial and theoretical approach was restricted to a subset of the possible Circus specifications: those dealing with only one same

\footnotetext{
3 https://bitbucket.org/circusmodelcheck/circus2csp
} 
type for all variables within the state of those processes. Thus, we had to implement not only a tool for the translation but also to refine that translation strategy in order to support a more realistic set of specifications: those using mixed types among their state variables.

We also experimented with the efficiency of FDR concerning the scale of the specifications. For such, we used the haemodialysis case study [123, a complex system which behaves according to the values of dozens of state variables. Thus, we refined the memory model in order to optimise the task of reading and updating the state variables from the Circus processes.

The outcome is that we now have a mechanised translator from Circus to $C S P_{M}$ that produces tractable models, and allows the use of FDR on larger case studies than have been possible up to now. The new developed approach, as described in this paper, is sound since we were able to prove, by hand as well as using FDR as a refinement checker, that the memory model from Oliveira et $a l$. is refined by the model discussed here [11, p. 77].

As part of the translation, we implemented an automatic refinement calculator for Circus2CSP, which handles a selected set of Circus refinement laws used according to 26, Appendix A, p. 147]. Moreover, we experimented with a strategy for refining Z schemas into "schema-free" Circus actions using Z Refinement Calculus [6].

Deliverables In summary, our research towards model checking Circus resulted in the following contributions:

- A tool for automatically translating a subset of Circus into $C S P_{M}$ : Implementation of a tool based on the work of Oliveira et al. 26] where one is able to translate Circus models written in $\mathrm{AT}_{\mathrm{E}} \mathrm{X}$ into $\operatorname{CSP}_{M}$, and then, be able to perform model-checking and refinement checks using FDR.

- An automatic Circus refinement calculator: As part of the translation strategy, the Circus refinement laws are applied to the processes and actions. In order to automate the translation as much as possible, we provide an automatic Circus refinement calculator.

- A transformation of some $\mathrm{Z}$ schemas into appropriate Circus constructs for translating into $C S P_{M}$ : The translation approach presented by Oliveira does not handle $\mathrm{Z}$ schemas directly, but only after normalisation. However, such a translation was not yet formally proved to be correct. We explore ways of translating $\mathrm{Z}$ schemas into Circus actions, specifically, those schemas where the translation results in a set of assignments.

- An improved Circus model that supports multiple types within a specification: The generated $C S P_{M}$ model from Oliveira et al. using multiple types is not supported by FDR, since it contains some auxiliary functions that are seen by FDR as polymorphic functions, which are not supported by such a tool. We, however, introduce a new data structure that treats each type with its own set of auxiliary functions.

- A refinement of the memory model from Oliveira et al. [26]: We provide a refined memory model with distributed memory cells updating and 
retrieving the values of the state variables, allowing FDR to handle a large number of state variables in a process, optimizing FDR's effort to check such models.

- New rules for mapping Circus to $C S P_{M}$ : We extended the mapping functions for expressions and predicates from $\mathrm{Z}$, as well as mapping functions for those actions specifically related to the Memory model.

- A mechanism that integrates Circus2CSP with FDR: We connected our tool to the "terminal-mode" interface of FDR, in order to be able to run checks straight from our tool. Unfortunately, we have no direct access to the code of FDR, and thus, we have to manually parse the results from the execution of FDR's "refine" command.

- An automatic assertion generator for checking with FDR: Our tool is able to generate assertion checks for refinement, deadlock, livelock and determinism checks for the loaded specification.

Tool Restrictions Our tool expects Circus specifications as input, written as a sequence of $\mathrm{IAT}_{\mathrm{E} X} \mathrm{X}$ block environments, very similar to the way $\mathrm{Z}$ paragraphs are written in $\mathrm{I}_{\mathrm{E}} \mathrm{X}$, which is a de facto standard for writing Circus specifications. However, we assume that the Circus document is already type checked by existing tools [21.

Our tool supports most of the Circus syntax, avoiding those constructs not handled in [26, p78] such as: no writting to input variables; external choice only among prefixed actions (those guaranteed to participate in an event before doing anything else, such as assignment); and no miraculous specifications.

Furthermore, some features are not yet supported such as: dealing with state invariants or preconditions in the $\mathrm{Z}$ schemas; non-determinism of data is not supported; and the consequences of nested parallelism and hiding with nondisjoint name sets have not been handled yet. These are a consequence of this being an automated translation, rather than the manual one prescribed in [26]. Finally, the translation of $\mathrm{Z}$ schemas used as Circus actions is restricted to those resulting in assignments.

\section{Experiments with Circus2CSP}

During our research we performed tests using our tool, Circus2CSP, exploring ways of overcoming any limitations from FDR, as well as comparing our approach with others from the literature.

Firstly, we explore the interference of invariants and preconditions in $\operatorname{CSP}_{M}$, using the chronometer model from Oliveira [25, p.34-41], comparing the model from Circus2CSP with the translation from Oliveira [26. We identified that using Circus2CSP the time spent by FDR to check for deadlock freedom, for example, with a model with the natural numbers ranging from zero to sixty $(0 . .60)$, was of around 3 minutes. However, using Oliveira's approach it took nearly three hours. In terms of states visited, FDR explored around 25 thousand states, compared with 99 million using the approach of Oliveira. In general, the $C S P_{M}$ models 
translated using our tool were evaluated by FDR using a much smaller state space and were checked in up to $95 \%$ less time than all the other models we tried derived from Oliveira's. However, we observed no correlation between time and state visited, in spite of the use (or not) of compression by default in FDR.

Then, we compare the translation of the haemodialysis case study using Circus2CSP with the Circus model from [12, which was manually translated into $C S P_{M}$ for model-checking. We observed a reduction of over $91 \%$ of the state explored, as well as the execution time. Moreover, the manual translation didn't allow us to run FDR with a larger range of values for natural numbers, usually ranging from 0 up to 2 . However, with Circus2CSP, we were able to go beyond the range 0 up to 90 in less than a minute. Such a result demonstrated that our approach is capable of handling large-scale case studies like the haemodialysis machine 12 and the ring buffer 3826 .

We also the effects of using some compression techniques available in FDR using the haemodialysis case study as an example. Although the states/transition$\mathrm{s} /$ plys visited were considerably reduced using the compression techniques such as sbisim, which determines the maximal strong bisimulation [5], and wbisim, which computes the maximal weak bisimulation, there was little impact on overall execution time, and the number of states visited are independent of the range of natural numbers used, while the number of transitions grows slowly. However, it is difficult to identify which compression technique will be most effective in a general case, and indeed, further experiments are required.

Finally, we compare different approaches for modeling the Ring Buffer case study 26 38, using FDR, in order to test the capabilities of our tool while modelchecking the translated models, in contrast to the limitations of ProB [19]. Unfortunately, the structure defined for our translation strategy is not fully supported by ProB, which was used to test the model generated with the translation strategy from Ye [38. ProB is another model-checker, which was originally developed for the B language, and was extended to support CSP, Z, Event-B [1], as well as combined languages such as CSP||B. We observed that some of the constructs used in our $C S P_{M}$ model, such as subtype, are not yet supported by ProB. Nevertheless, we were able to use ProB's animator and to execute the same assertion check, as in FDR, obtaining similar results.

However, the tests performed with the $C S P_{M}$ specification of Ye using FDR failed to checks for deadlock freedom and determinism. The results obtained from ProB can be related to what we obtained in FDR in terms of the behavior of the system: the counterexample given from FDR can be used to animate the CSP I IB model in ProB, causing the same effect: deadlock. Although, our experiment was limited since CSP I | B takes into account the system state in ProB. In such model, the $C S P_{M}$ file generated from Ye captures only the behavior of the system, but does not captures the system state. We reckon that the deadlock was caused because the state (modeled in B) can interfere in the system behavior in order to avoid deadlocks. 


\section{Conclusions}

In this paper, we briefly introduced Circus2CSP, a tool capable of model-checking Circus specifications using FDR, through a translation strategy from Circus into $C S P_{M}$. It comprises a series of translation rules, combined with Circus refinement laws. One can perform refinement checks using FDR directly from Circus2CSP's command-line. The tool can be downloaded freely from https://bitbucket. org/circusmodelcheck/.

We improved Oliveira's [26] translation strategy in a few ways: handling a wider mix of datatypes; translating Z schemas easily "compiled" to assignments; coping better with potentially large state spaces; and close integration with FDR. Some of the equivalence laws used in the translation have side-conditions that lead to proof obligations. Our tool does not discharge these, leaving them to the user to handle by other means.

The modifications for the memory model developed for our tool are similar to what was presented by Mota et al. 24, where interleaving between processes, one for each state variable, was proposed. In fact, the memory model used in [26] was based on the one by Mota et al., and was expanded with the inclusion of a terminate signal, and, rather than one process for each variable, it would offer all possible mget and mset for all state variables at the same time.

However, after implementing Oliveira's memory model, we identified that such an approach tends to make the job of FDR harder and expensive, leading to state space explosion, when using several state variables on the same memory model. In our research, we also seek ways of optimising the ${ }_{C S P}$ model in order to obtain a more efficient analysis. Therefore, even though the terminate event is used for synchronising the end of the Memory execution, the parallel composition of all possible mgets and msets leads to a possible exponential growth of the state space. However, such a problem does not occur while using interleaving. Moreover, Mota et al. also argues that the use of interleaving helps the compression algorithms built in FDR [30] to reduce the state space exploration while analysing such models.

A key principle in critical software development methods is that all global variables should be intialised pretty much immediately [2]. In a Circus context, if all the assignments are done are before any observable event occurs, then its behaviour is that of a (simultaneous) assignment $s^{\prime}=s_{\text {init }}$, where $s$ is the (aggregated) global state. This allows us to introduce an additional translation step that replaces a non-deterministic choice over all possible starting values of $s$ by one arbitrary choice of starting value for $s$. This is normally a proper refinement, but with initialisation as above, results in being an equivalence. This trick dramatically improved the performance of FDR.

Some related work on techniques for model-checking Circus was presented by Freitas 8 . where a refinement model checker based on automata theory [16] and the operational semantics of Circus [35] was formalised in Z/Eves [31. He also prototyped a model checker in Java. However, Freita's Circus model checker is restricted to a subset of Circus actions and does not support the notion of Circus processes. Moreover, Nogueira et al. 23] also presented a prototype of a 
model checker based on the operational semantics of Circus within the Microsoft FORMULA [18 framework. However, they could not provide a formal proof of the soundness of their approach, since FORMULA does not have an available formal semantics.

Yet another approach for model-checking Circus was defined by Ye and Woodcock [37, who defined a link from Circus to $C S P \| B$ with model-checking using ProB [28. However, ProB is a limited tool in terms of processing capabilities: it does not support multiprocessors nor multithreading, and therefore, it is unlikely that it would support larger models such as those checked using Circus2CSP. Moreover, the translation proposed by Ye produces a specification split in two languages: B for the state machine and CSP for the behaviour of the model. However, such an approach is limited to be checked by ProB while ours isn't limited to FDR: we can indeed load our $C S P_{M}$ translated models in ProB, and still perform model-checking. Finally, Beg [4] prototyped and investigated an automatic translation that supports a subset of Circus constructs, supporting only Skip, prefixing action, sequential composition, assignments, if statements, and guards with simple predicates.

For future work, we have plans for specifying a translation strategy for Z schemas used as Circus actions within a process. The best approach would be to use Z Refinement Calculus [6. For now, our tool deals only with those schemas that in fact can be translated into assignments. We intend to explore the operators for $\mathrm{Z}$ schemas and the refinement laws that can be applied accordingly.

Acknowledgments This work was funded by CNPq (Brazilian National Council for Scientific and Technological Development) within the Science without

Borders programme, Grant No. 201857/2014-6, and partially funded by Science Foundation Ireland grant 13/RC/2094.

\section{References}

1. Abrial, J.R.: Modeling in Event-B: System and Software Engineering. Cambridge University Press, New York, NY, USA, 1st edn. (2010)

2. Aeronautical Radio, I.A.: ARINC 653: Avionics Application Standard Software Interface (nov 2006)

3. Atif, M.: The hemodialysis machine case study. In: Butler, M.J., Schewe, K.D., Mashkoor, A., Biró, M. (eds.) Lecture Notes in Computer Science (including subseries Lecture Notes in Artificial Intelligence and Lecture Notes in Bioinformatics). Lecture Notes in Computer Science, vol. 9675, pp. 329-343. Springer (2016), https://doi.org/10.1007/978-3-319-33600-8_29

4. Beg, A., Butterfield, A.: Development of a prototype translator from Circus to CSPm. In: ICOSST 2015 - 2015 International Conference on Open Source Systems and Technologies, Proceedings. pp. 16-23 (dec 2016)

5. Boulgakov, A., Gibson-Robinson, T., Roscoe, A.W.: Computing maximal weak and other bisimulations. Formal Aspects of Computing 28(3), 381-407 (2016), https://doi.org/10.1007/s00165-016-0366-2 
6. Cavalcanti, A., Woodcock, J.C.P.: ZRC - A Refinement Calculus for Z. Formal Aspects of Computing 10(3), 267-289 (mar 1998), http://link.springer.com/ 10.1007/s001650050016https://doi.org/10.1007/s001650050016

7. Dijkstra, E.W.: Guarded commands, nondeterminacy and formal derivation of programs. Communications of the ACM 18(8), 453-457 (1975), http://portal.acm. org/citation. $\mathrm{cfm}$ ?doid $=360933.360975\{\%\} 5 \mathrm{Cn}$

8. Freitas, L.: Model checking Circus. Ph.D. thesis, Department of Computer Science, The University of York, UK (2005)

9. Gibson-Robinson, T., Armstrong, P., Boulgakov, A., Roscoe, A.W.: FDR3 - A Modern Model Checker for CSP. Tools and Algorithms for the Construction and Analysis of Systems 8413, 187-201 (2014), http://www.cs.ox.ac.uk/projects/ fdr/manual/

10. Gomes, A.O.: Formal Specification of the ARINC 653 Architecture Using Circus (2012), http://etheses.whiterose.ac.uk/id/eprint/2683

11. Gomes, A.O.: Model-Checking Circus with FDR using Circus2CSP. Ph.D. thesis, Trinity College Dublin (2019), http://www.tara.tcd.ie/handle/2262/86009

12. Gomes, A.O., Butterfield, A.: Modelling the haemodialysis machine with Circus. Lecture Notes in Computer Science (including subseries Lecture Notes in Artificial Intelligence and Lecture Notes in Bioinformatics) 9675(201857), 409-424 (2016), https://doi.org/10.1007/978-3-319-33600-8_34

13. Gomes, A.O., Butterfield, A.: Circus2CSP - A Translator from Circus to CSPm (2018), https://bitbucket.org/circusmodelcheck/circus2csp

14. Gomes, A.O., Butterfield, A.: Towards a Model-Checker for Circus. In: In This Volume. Springer Berlin Heidelberg, Berlin, Heidelberg (2019)

15. Hoare, C., He, J.: Unifying Theories of Programming. Prentice-Hall (1998)

16. Hopcroft, J.E., Motwani, R., Ullman, J.D.: Introduction to automata theory, languages, and computation - international edition (2. ed). Addison-Wesley (2003)

17. Hopkins, D., Roscoe, A.W.: SVA, a tool for analysing shared-variable programs. Electronic Notes in Theoretical Computer Science pp. 1-5 (2007), http://www.cs . ox.ac.uk/people/bill.roscoe/publications/119.pdf

18. Jackson, E.K., Levendovszky, T.T., Balasubramanian, D.: Reasoning about metamodeling with formal specifications and automatic proofs. In: Whittle, J., Clark, T., Kühne, T. (eds.) Lecture Notes in Computer Science (including subseries Lecture Notes in Artificial Intelligence and Lecture Notes in Bioinformatics). Lecture Notes in Computer Science, vol. 6981 LNCS, pp. 653-667. Springer (oct 2011), https://doi.org/10.1007/978-3-642-24485-8_48

19. Leuschel, M., Butler, M.: ProB: An automated analysis toolset for the B method. International Journal on Software Tools for Technology Transfer 10(2), 185-203 (2008)

20. Lowe, G.: Specification of communicating processes: Temporal logic versus refusalsbased refinement. Formal Aspects of Computing 20(3), 277-294 (2008), https: //link. springer. com/content/pdf/10.1007\{\%\}2Fs00165-007-0065-0.pdf

21. Malik, P., Utting, M.: CZT: A Framework for Z Tools. ZB 2005: Formal Specification and Development in Z and B 3455, 315-352 (2005), http://czt.sourceforge.nethttps://doi.org/10.1007/11415787_5http: //dx.doi.org/10.1007/11415787-5

22. Morgan, C., Carroll: Programming from specifications (2nd. Ed.), Prentice Hall International series in computer science, vol. 16. Prentice Hall (1994), https:// dl.acm.org/citation. cfm?id=184737 
23. Mota, A., Farias, A.C., Didier, A., Woodcock, J.C.P.: Rapid prototyping of a semantically well founded Circus model checker. In: Giannakopoulou, D., Salaün, G. (eds.) Lecture Notes in Computer Science (including subseries Lecture Notes in Artificial Intelligence and Lecture Notes in Bioinformatics). Lecture Notes in Computer Science, vol. 8702 LNCS, pp. 235-249. Springer (2014), https: //doi.org/10.1007/978-3-319-10431-7_17

24. Nogueira, S., Sampaio, A., Mota, A.: Test generation from state based use case models. Formal Aspects of Computing 26(3), 441-490 (2014)

25. Oliveira, M.V.M.: Formal Derivation of State-Rich Reactive Programs using Circus. Ph.D. thesis, University of York, UK (2005), http://ethos.bl.uk/ OrderDetails.do?uin=uk.bl.ethos.428459

26. Oliveira, M.V.M., Sampaio, A., Antonino, P., Ramos, R., Cavalcanti, A., Woodcock, J.C.P.: Compositional Analysis and Design of CML Models. Tech. Rep. D24.1, COMPASS Deliverable (2013), http://www.compass-research.eu/ Project/Deliverables/D241.pdf

27. Oliveira, M.V.M., Sampaio, A., Conserva Filho, M.: Model-Checking Circus StateRich Specifications. Integrated Formal Methods 20148739 LNCS, 39-54 (2014), https://doi.org/10.1007/978-3-319-10181-1_3

28. Plagge, D., Leuschel, M.: Validating Z Specifications Using the ProB Animator and Model Checker. In: Davies, J., Gibbons, J. (eds.) Integrated Formal Methods, Lecture Notes in Computer Science, vol. 4591, pp. 480-500. Springer (2007), https: //doi.org/10.1007/978-3-540-73210-5_25

29. Roscoe, A.W.: The Theory and Practice of Concurrency. Prentice Hall PTR, Upper Saddle River, NJ, USA (1973)

30. Roscoe, A.W.: Understanding Concurrent Systems, Texts in Computer Science, vol. 42. Springer London, London (2010), http://www.ncbi.nlm.nih.gov/pubmed/ 20549881

31. Saaltink, M., Meisels, I., Saaltink, M.: The Z/EVES Reference Manual (for version 1.5). Reference manual, ORA Canada pp. 72-85 (1997), http://dl . acm.org/ citation. $c f m ?$ id $=647282.722913$

32. Schneider, S.: Concurrent and Real-time systems. Wiley Chichester, UK (2000)

33. Utting, M.: Jaza User Manual and Tutorial (jun 2005)

34. Woodcock, J.C.P., Cavalcanti, A.: The Semantics of Circus. In: Zb 2002: Formal Specification and Development in Z and B: 2nd International Conference of B and Z Users Grenoble (2002)

35. Woodcock, J.C.P., Cavalcanti, A.L.C., Freitas, L.: Operational Semantics for model checking Circus. Lecture Notes in Computer Science 3582, 237-252 (2005)

36. Woodcock, J.C.P., Davies, J.: Using Z, Specification, Refinement, and Proof. Prentice Hall International Series in Computer Science, Prentice-Hall, Inc., Upper Saddle River, NJ, USA (1996)

37. Ye, K.: Model Checking of State-Rich Formalisms. Ph.D. thesis, University of York (2016)

38. Ye, K., Woodcock, J.C.P.: Model checking of state-rich formalism Circus by linking to CSP_B. International Journal on Software Tools for Technology Transfer 19(1), 73-96 (2017), https://doi.org/10.1007/s10009-015-0402-1 